\title{
MS03-P14 | One Step Co-Purification And Crystallization Of Three Soluble Proteins From Cyanobacteria, The Unique Crystallization Properties Of C-
}

PhyCOCYANIN

Sarrou, losifina (CFEL, DESY, Hamburg, GER); Falke, Sven (Institute for Biochemistry, Hamburg, GER); Komadina, Dana (CFEL, DESY, Hamburg, GER); Tritschler, Felix (CFEL, DESY, Hamburg, GER); Yefanov, Oleksandr (CFEL, DESY, Hamburg, GER); Chapman, Henry (CFEL, DESY, Hamburg, GER)

Cyanobacteria have gained a lot of attention in recent years because of their potential applications in various fields of biotechnology. The establishment of cost and time-efficient purification protocols for cyanobacterial proteins and complexes, especially from their natural source, is highly desirable in structural biology and other areas of research.

We are presenting a highly time-efficient optimized co-purification protocol for three native key proteins of the photosynthetic cyanobacteria T. elongatus and A. platensis:

The cytochrome c6, a redox protein acting as an electron mediator between the cytochrome b6f complex and photosystem I. The 2Fe-2S cluster Ferredoxin (Fed1) which is reduced by Photosystem I and carries one electron to a Fd-NADP+ reductase. The antenna protein C- Phycocyanin (C-PC), a pigment with massive commercial value as natural colorants in nutraceutical, cosmetics, and pharmaceutical industries, besides its health benefits.

As obtained, we were able to characterize and optimize crystallization conditions for cytc6 and Fed1 from $T$. elongatus and collected X-ray diffraction data from crystals with resolutions higher than $2 \AA$.

Further, C-Phycocyanin from T. elongatus and A. platensis show unique crystallization properties. They crystallize in hundreds of significantly different conditions with various crystal morphologies. We collected high resolution data from 160 different crystallization conditions in the $\mathrm{pH}$ range 4 to 9 and will present the changes in the symmetry and the unit cell parameters under different conditions. This extraordinary behaviour of the C-PC will contribute to the comprehension of the fundamental principles of protein crystallography. 